\title{
Infrared Analysis of Thin Films: Amorphous, Hydrogenated Carbon on Silicon
}

\author{
Wolfgang Jacob, Achim von Keudell, and Thomas Schwarz-Selinger \\ Max-Planck-Institut für Plasmaphysik, \\ EURATOM Association, Boltzmannstr.2, 85748 Garching, Germany
}

Received 28 February, 2000

\begin{abstract}
The infrared analysis of thin films on a thick substrate is discussed using the example of plasmadeposited, amorphous, hydrogenated carbon layers $(\mathrm{a}-\mathrm{C}: \mathrm{H})$ on silicon substrates. The framework for the optical analysis of thin films is presented. The main characteristic of thin film optics is the occurrence of interference effects due to the coherent superposition of light multiply reflected at the various internal and external interfaces of the optical system. These interference effects lead to a sinusoidal variation of the transmitted and reflected intensity. As a consequence, the Lambert-Beer law is not applicable for the determination of the absorption coefficient of thin films. Furthermore, observable changes of the transmission and reflection spectra occur in the vicinity of strong absorption bands due to the Kramers-Kronig relation. For a sound data evaluation these effects have to be included in the analysis. To be able to extract the full information contained in a measured optical thin film spectrum, an experimentally measured spectrum has to be simulated using the full formalism including the Kramers-Kronig relation. Infrared absorption spectra and the resulting $\mathrm{k}$ spectra in the range of the $\mathrm{CH}$ vibrational bands around $3000 \mathrm{~cm}^{-1}$ are presented for a variety of a-C:H layers. The shape and the total intensity of the peak are quite sensitive to the film structure. Soft, polymerlike hydrocarbon layers are characterized by a well structured, intense IR absorption band, while hard, amorphous, hydrogenated carbon layers exhibit a structureless, broad IR absorption band with relative low intensity. The $\mathrm{k}$ spectra of the $\mathrm{CH}$ vibrational bands can be considered as fingerprint for the type of a-C:H film.
\end{abstract}

\section{Introduction}

Infrared absorption spectroscopy is a wide-spread and easily accessible technique. Above that, infrared absorption spectroscopy is nondestructive and a matured field of materials analysis which is well covered in large number of textbooks and monographs [1,2]. In the following the acronym IRAS will be used for infrared absorption spectroscopy. This acronym should not be confused with IRRAS which means infrared reflection absorption spectroscopy and which is usually performed under grazing incidence for monolayer films on a metallic substrate.

Many laboratories world wide have easy access to IRAS instruments and no laborious sample preparation is necessary if thin films are directly deposited on an infrared-transparent substrate, such as, for example, single crystalline silicon. Due to the combination of the mentioned favorable experimental prerequisites IRAS is often applied for the analysis of thin films. In many such cases silicon is anyway used or can easily be used as substrate material. If not, other infrared-transparent materials can be found or the measurement can be performed in reflection mode or applying attenuated total reflection [2-4]. But besides these more practical arguments, there are sound physical arguments that speak for IRAS as a method for thin film analysis. IRAS is not only capable of delivering qualitative structural information about the bulk of the deposited material, but can also, under favorable circumstances, yield quantitative results of the density of certain infrared-active structural groups or even of the layer stoichiometry. A good example for the latter is the analysis of plasmadeposited, amorphous, hydrogenated silicon layers (a$\mathrm{Si}: \mathrm{H})$. This is based on the basic investigations of Brodsky et al. [5] who have shown that the hydrogen content of a-Si:H can be determined by IRAS. Later it was shown by Langford et al. [6] that the method of Brodsky et al., although in principal correct, can lead to errors of the order of $75 \%$ in the quantitative analysis. These errors were mainly attributed to the occurrence of coherent multiple reflections in the film, which lead to an increase of the effective optical path length. The quantitative analysis of IRAS spectra as demonstrated for a-Si:H can not simply be generalized and the physical requirements for it have to be carefully checked in each case. For example, in the above case of a-Si:H, the quantitative evaluation is based on the fact that 
the transition dipole moments ('dipole strength') of the different $\mathrm{Si}-\mathrm{H}$ stretching vibrations, which contribute to the absorption band generally used for analysis have been individually determined by comparison with other quantitative methods [5,6]. It has to be stressed, that the transition dipole moments of individual IR bands can differ significantly, by up to more than an order of magnitude. The analysis of the IR spectra of amorphous, hydrogenated carbon (a-C:H) films is, however, often based on the assumption that all vibrations contributing to the $\mathrm{CH}$ stretch vibrations located around $3000 \mathrm{~cm}^{-1}$ are of the same magnitude. This led to many wrong and ambiguous results in the case of a-C:H [7-9].

But before such question can be discussed on a sound basis, another point has to be considered. This is the general analysis of IRAS spectra from thin films. Many authors simply use the Lambert-Beer law [10,11] which predicts an exponential decrease of the transmitted intensity with increasing film thickness. This simple analysis is incorrect in the case of thin films because it takes not into account interference effects of the investigated optical system. The same was already pointed out by Langford et al. in the case of a-Si:H [6]. A thin film on a substrate differs considerably from typical IRAS setups described in textbooks where fluids, gases, or rather thick solids are investigated. Another related problem is the correct treatment of the background. Both of these points will be discussed in the first section of this article.

The article is organized as follows: in the first section, the theoretical framework for optical analysis of transparent thin films on a substrate is presented. This framework takes into account interference effects due to multiple reflections at the boundaries of the optical system and the contribution of absorption peaks to the real part of the refractive index according to the Kramers-Kronig relation, which slightly modifies the background under an absorption peak. The second section presents some results of the infrared analysis of plasma-deposited, amorphous, hydrogenated carbon films (a-C:H) and a comparison to quantitative results such as the refractive index or the stoichiometry

\section{Optical analysis of thin films}

The problem we are dealing with is the analysis of the response of an optical system to incident electromagnetic radiation. In this context, the following processes play a role: reflection, transmission, absorption, and interference. This is treated in great detail in a large number of textbooks on optics [10,12]. A full, rigorous treatment of the electromagnetic field equations may be found in the textbook of Born and Wolf [12]. The application of the same principles to thin film filters is treated by Macleod [13]. An excellent discussion of the theoretical framework with respect to thin films can be found in the book of Stenzel [11] which is, however, only available in German. A highly condensed English version of the same material is found in Ref. [14]. The theoretical formalism is further treated by Harbecke and coworkers [15-18].

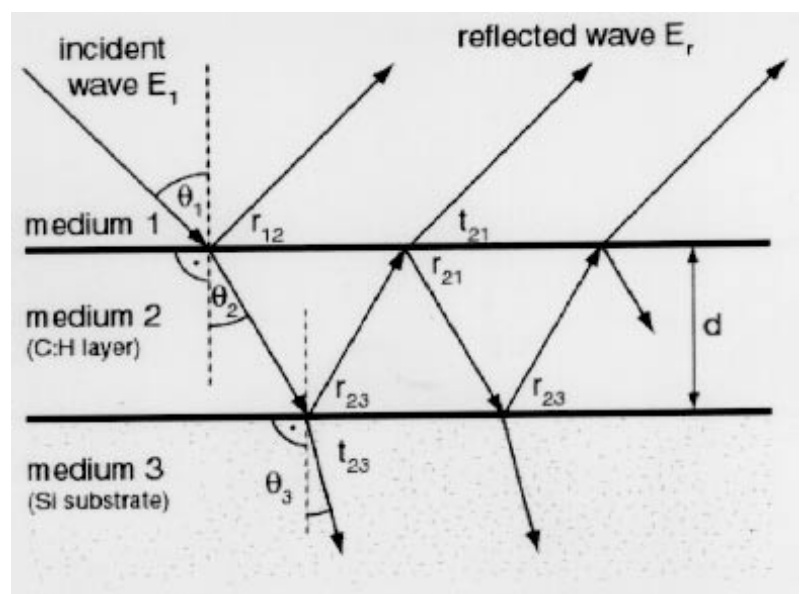

Figure 1. Schematic representation of the investigated optical system: a thin film on top of a thick substrate. The boundary on the second side of the substrate is not shown. In general, the medium behind the substrate is identical to medium 1 .

The underlying analysis is in principle the same irrespective of whether we perform transmission or reflection measurements or if we measure the change in the state of polarization as in ellipsometry $[19,20]$. The analysis in the following will be made for a simple transmission measurement of a thin film on top of an infrared-transparent substrate. The most simple conceivable system is that of a thin homogeneous layer on top of a thick substrate. The system is sketched in Fig. 1. Both, substrate and layer are characterized by their complex refractive index $N=n-i k$. We will use this notation throughout this article. $N$ stands for the complex refractive index, $n$ is the real and $k$ the imaginary part of the refractive index. $k$ is also often denoted as extinction coefficient. The thin layer is further characterized by its thickness $d$. In real cases, the situation may sometimes be a bit more complicated due to the presence of a thin interfacial layer of either native silicon dioxide or a modified layer due to a possible in-situ cleaning step prior to deposition such as, for example, sputtering. This can easily be included in the analysis applying a similar procedure as presented in this article, but will not be further worked out here.

If a beam of light hits the interface of two media it will be partially reflected (subscript $\mathrm{r}$ ) and partially transmitted (refracted) (subscript t). Let $n_{1}$ be the real part of the refractive index of medium 1 and $n_{2}$ that of medium 2. $\theta_{1}$ and $\theta_{2}$ are the angles between the surface normal and the incident and the transmitted beams, respectively, as indicated in Fig. 1. The relation between $\theta_{1}$ and $\theta_{2}$ is given by Snell's law [see 10-13]: 


$$
n_{1} \sin \theta_{1}=n_{2} \sin \theta_{2}
$$

Let further be $E_{1}$ the field vector of the incident beam. $E_{1}$ is split into 2 components $E_{p}$ and $E_{s}\left[E_{1}=\right.$ $\left.\left(E_{p, 1}, E_{s, 1}\right)\right]$, which are parallel $(p)$ and perpendicular $(s)$ to the plane of incidence. Then the amplitude reflection and transmission coefficients $r_{p}, r_{t}$ and $t_{p}, t_{s}$ are given by Fresnel's equations [see 10-13]. The Fresnel coefficients describe the ratios of the amplitudes of the respective beams to the corresponding amplitude of the incident beam (e.g., $r_{p}=E_{p, r} / E_{p, 1} ; r_{p}=$ reflection coefficient for the parallel component of $E ; E_{p, r}$ and $E_{p, 1}$ being the parallel components of the reflected and incident electric field, respectively).

$$
\begin{aligned}
& r_{p}=\frac{N_{2} \cos \theta_{1}-N_{1} \cos \theta_{2}}{N_{2} \cos \theta_{1}+N_{1} \cos \theta_{2}} \\
& r_{s}=\frac{N_{1} \cos \theta_{1}-N_{2} \cos \theta_{2}}{N_{1} \cos \theta_{1}+N_{2} \cos \theta_{2}} \\
& t_{p}=\frac{2 N_{1} \cos \theta_{1}}{N_{2} \cos \theta_{1}+N_{1} \cos \theta_{2}} \\
& t_{p}=\frac{2 N_{1} \cos \theta_{1}}{N_{1} \cos \theta_{1}+N_{2} \cos \theta_{2}}
\end{aligned}
$$

where the subscripts $p$ and $r$ denote the parallel $(p)$ and perpendicular $(s)$ components. In the general case, these coefficients are complex numbers. As intensities are proportional to the square of the complex field amplitudes, the corresponding intensity reflection coefficients are given by $R_{12}=r_{12} r_{12}^{*}$, and so on, where $r^{*}$ denotes the complex conjugate. The notation $R_{i j}$ means the reflection coefficient for light incident from medium 1 on the interface to medium 2. The transmission coefficient $T_{12}$ is given by $1-R_{12}$. It should be kept in mind that $T_{12}$ is, due to it's definition by the Poynting vector $[11,12]$, not equal to $t_{12} t_{12}^{*}$, but:

$$
T_{12}=1-R_{12}=\frac{N_{2} \cos \theta_{2}}{N_{1} \cos \theta_{1}} t_{12} t_{12}^{*}
$$

Due to the multiple reflections at the internal interfaces a part of the incident light intensity traverses the thin film several times corresponding to an increase of the effective optical path length. We get, therefore, a analytical dependence that differs significantly from the well known Lambert-Beer law:

$$
I(x)=I_{0} \exp (-\alpha x) .
$$

The Lambert-Beer law describes the attenuation of light travelling through isotropic, homogeneous matter. $I(x)$ is the remaining intensity at position $x, I_{0}$ is the incident intensity (at position $x=0$ ), and $\alpha$ is the absorption coefficient. $\alpha$ is related to the imaginary part of the refractive index (the extinction coefficient $\mathrm{k}$ ) of the medium as following:

$$
\alpha=\frac{4 \pi k}{\lambda}
$$

It is common to define the reflectance $R$ of the sample as the ratio of the specularly reflected intensity $I_{r}$ to the incident intensity $I_{i}\left(R=I_{r} / I_{i}\right)$ and the transmittance $T$ as $T=I_{t} / I_{i}$, the absorptance $A$ as $A=I_{a} / I_{i}$, and the optical scatter $S$ as the ratio of the diffusely scattered intensity to the incident intensity $\left(S=I_{s} / I_{i}\right)$. The reflectance and transmittance are often also named reflectivity and transmittivity, respectively (For name conventions see Ref. 4, page 50). Energy conservation requires:

$$
R+T+A+S=1 .
$$

In many practical applications, in particular the ones considered here, the optical scatter is negligible and Eq. 4 simplifies to $R+T+A=1$.

As mentioned above, it is, for a thin-film system, not appropriate to use Lambert-Beer law because multiple reflections in the thin film have to be considered, as indicated in Fig. 1. The reflected intensity is composed of an infinite number of individual, multiply reflected beams. In the case of a non-absorbing layer (i.e., $N_{2}$ is real, $N_{2}=n_{2}$ ) on a semi-infinite substrate (this means that no light returns from the backside of the substrate), we can write the reflectance $R$ as:

$$
\begin{aligned}
R & =R_{12}+T_{12} R_{23} T_{21}+T_{12} R_{23} R_{21} R_{23} T_{21}+T_{12} R_{23}\left(R_{21} R_{23}\right)^{2} T_{21}+ \\
& =R_{12}+T_{12} R_{23} T_{21} \sum_{j=1}^{\infty}\left(R_{21} R_{23}\right)^{j-1}=R_{12}+\frac{T_{12} R_{23} T_{21}}{1-R_{21} R_{23}}
\end{aligned}
$$


And the transmittance is given by:

$$
T=1-R
$$

It should be mentioned here that the summation of the intensities corresponds to the incoherent superposition of the individual light beams as opposed to the coherent superposition discussed further below.

Let us consider now the common case of perpendicular incidence ( $\theta_{1}$ and $\left.\theta_{2}=0\right)$ and medium 1 being air $\left(N_{1}=1-i 0\right)$. Lets in addition assume that we are dealing with a free standing film, i.e. medium 3 is also air $\left(N_{3}=N_{1}\right)$, then $R_{23}$ changes to $R_{21}$. Using the identity $R_{12}=R_{21}$, Eqs. 7 and 8 simplify to:

$$
\begin{gathered}
R=\frac{2 R_{12}}{1+R_{12}}=\frac{\left(n_{2}-1\right)^{2}}{n_{2}^{2}+1} \\
T=\frac{1-R_{12}}{1+R_{12}}=\frac{2 n_{2}}{n_{2}^{2}+1}
\end{gathered}
$$

The above considerations leading to Eqs. 7 to 10 were made for a non-absorbing medium. For an absorbing medium Eqs. 7 to 10 can be generalized by replacing each occurrence of $R_{12}, R_{21}$ and $T_{21}$ in the derivation of Eq. 7 according to:

$$
T_{21} \rightarrow T_{21} e^{-\alpha d} \text { rmand } R_{12(21)} \rightarrow R_{12(21)} e^{-\alpha d} .
$$

The term $e^{-\alpha d}$ accounts for the loss of intensity in a single path through our layer with thickness $d$. $\alpha$ is the absorption coefficient that we already know from the Lambert-Beer law (Eq. 4). This replacement yields (we again use $R_{12}=R_{21}$ ):

$$
R=\frac{R_{12}\left[1-e^{-2 \alpha d}\left(2 R_{12}-1\right)\right]}{1-R_{12}^{2} e^{-2 \alpha d}}
$$

and

$$
T=\frac{\left(1-R_{12}\right)^{2} e^{-\alpha d}}{1-R_{12}^{2} e^{-2 \alpha d}}
$$

As a consequence of the multiple reflections within our thin layer, the transmittance of our system given by Eq. 12 is not proportional to $e^{-\alpha d}$ as would be anticipated from a simple Lambert-Beer type behavior according to Eq. 4. The transmittance is only then approximately proportional to $e^{-\alpha d}$, if the reflection coefficients of both interfaces $\left(R_{12}\right.$ in the above case) are low. We should retain here, that the application of the Lambert-Beer law is, in general, not valid in the case of thin film optics. Unfortunately, it has, however, to be stated that it is still frequently applied in the literature.

The discussion so far was made on the basis of the involved light intensities. This is correct only, if the films are thicker than the coherence length of the light. If the film thickness becomes much smaller than the coherence length, we cannot simply add the intensities of the individual light beams to get the total intensity (as done in the derivation of Eqs. 7 to 12), but we have to add the amplitudes of the electric field strength observing the actual phase. This leads to the appearance of interference effects $[11,12,14]$ in the reflected and transmitted signals. The derivation of the formulas for the reflectance and transmittance is very similar to the derivation of Eq. 7. But instead of the intensity coefficients $R_{i j}$ and $T_{i j}$, we use the amplitude coefficients $r_{i j}$ and $t_{i j}$, and we have to multiply with the phase factor $e^{-i \beta}$ for each traversal of the layer. For the system depicted in Fig. 1, we can write down the amplitude reflection and transmission coefficients in close analogy to Eq. 7:

$$
\begin{aligned}
r_{123} & =r_{12}+t_{12} e^{-i \beta} r_{23} e^{-i \beta} t_{21}+t_{12} e^{-i \beta} r_{23} e^{-i \beta} r_{21} e^{-\beta} r_{23} e^{-i \beta} t_{21}+\ldots \\
& =r_{12}+t_{12} r_{23} t_{21} e^{-2 i \beta} \sum_{j=1}^{\infty}\left(r_{21} r_{23} r_{12} e^{-2 i \beta}\right)^{j-1}=r_{12}+\frac{t_{12} r_{23} t_{21} e^{-2 i \beta}}{1-r_{21} r_{23} e^{-2 i \beta}}
\end{aligned}
$$

Using the identities $t_{12} t_{21}=1-r_{12}^{2}$ and $r_{21}=-r_{12}$ which follow from Fresnel's equations, we can simplify Eq. 13 to:

$$
r_{123}=\frac{r_{12}+r_{23} e^{-2 i \beta}}{1+r_{12} r_{23} e^{-2 i \beta}}
$$

Correspondingly we find:

$$
t_{123}=\frac{t_{12} t_{23} e^{-i \beta}}{1+r_{12} r_{23} e^{-2 i \beta}}
$$

The phase $2 \beta$ follows from a simple geometrical consideration of the phase difference which is given by the path difference of two neighboring interfering light beams:

$$
\beta=\frac{2 \pi}{\lambda} d \sqrt{n_{2}^{2}-\sin ^{2} \theta_{1}}=\frac{2 \pi}{\lambda} d n_{2} \cos \theta_{2}
$$


The above formulas (Eq. 14 and 15) are valid for both polarizations. The reflectance and transmittance of the optical system is again given by:

$$
R_{123}=r_{123} r_{123}^{*} \text { and } T_{123}=\frac{N_{x} \cos \theta_{3}}{N_{1} \cos \theta_{1}} t_{123} t_{123}^{*}
$$

$R_{123}$ and $T_{123}$ describe the reflectance and transmittance through the system depicted in Fig. 1, a thin layer on top of a semi-infinite substrate. We will further on call this treatment the coherent description of the multiple reflections as opposed to the incoherent description in Eqs. 7 to 12. It is worth noting that the incoherent description yields the same results as setting the real part of the phase coefficient $\beta$ to zero, as the phase relation is destroyed between the multiple reflections, and adding the individual intensities of every reflection [15-18]. It can be shown that $e^{-\alpha d}=e^{+2 \operatorname{Im}(\beta)}$.

In reality, the substrate has a finite thickness and introduces another interface. In most cases the medium on the backside of the wafer (medium 4) is identical to medium 1. If the backside of the substrate is also of optical quality, as we so far implicitly assumed for all involved interfaces, we will also get reflection from the backside and we have to include this in our analysis. We use in our experiments in general silicon wafers which are polished on both sides, to get a higher energy throughput through the wafer and, thus, a higher sensitivity. We have, therefore, to include these reflections in the silicon wafer in our analysis too. If the backside is rough, reflections from the backside of the silicon wafer can be omitted. The thickness of the silicon wafer is, in general, so thick, that these reflections have to be treated incoherently, according to Eqs. 11 and 12 .

In practice, this is done by inserting $R_{123}, R_{321}$, and $T_{123}, T_{321}$ in Eqs. 11 and 12 instead of $R_{12}, R_{21}$, and $T_{12}, T_{21}$, respectively. Finally, in real samples, a thin interfacial layer of either native silicon dioxide or a modified layer due to a possible in-situ cleaning step prior to deposition such as, for example, sputtering, may be present between the deposited film and the substrate. This can be included in the analysis in a similar manner, but is not explained further here.

The total transmittance and reflectance through our system is then given by:

$$
\begin{array}{r}
T_{\text {total }}=\frac{T_{123} T_{34} \exp \left[+2 \operatorname{Im}\left(\beta_{3}\right)\right]}{1-R_{34} R_{321} \exp \left[+4 \operatorname{Im}\left(\beta_{3}\right)\right]} \\
R_{\text {total }}=R_{123} \frac{T_{123} T_{321} R_{34} \exp \left[+4 \operatorname{Im}\left(\beta_{3}\right)\right]}{1-R_{34} R_{321} \exp \left[+4 \operatorname{Im}\left(\beta_{3}\right)\right]}
\end{array}
$$

with $\beta_{3}$ being the phase shift occurring in medium 3 (in fact, for consistency reasons $\beta$ in Eqs. 13 to 15 should be replaced by $\beta_{2}$ and correspondingly $d$ by $d_{2}$, since they apply to medium 2).

These expressions allow to model the transmission through an uncoated as well as to a coated sample. They are used to model the transmission through the uncoated reference sample (the silicon wafer) $T_{\text {ref }}$ and the coated sample $T_{\text {film }}$. In a standard IRAS measurement the transmittance $T_{\exp }$ is measured relative to a reference sample (in general, a uncoated silicon substrate), so that the experimental transmittance is given by $T_{\exp }=T_{\text {film }} / T_{\text {ref }}$. By applying the above formalism $T_{\exp }$ can be directly compared to the model results $T_{\bmod }=\left(T_{\text {film }} / T_{\text {ref }}\right)_{\bmod }$. The parameters for the model calculation are the complex refractive indices and thicknesses of the individual layers. The known parameters are the optical constants and the thickness of the silicon substrate and interface layer, and, in general, the thickness of the deposited layer. They can be taken from literature or determined by other methods such as ex-situ ellipsometry and profilometry. As unknown parameter remains the complex refractive index $N_{\text {film }}=n_{\text {film }}-i k_{\text {film }}$ of the deposited layer. These two parameters are determined by a computer-based fitting routine which fits the model spectrum $T_{\bmod }$ to the measured spectrum $T_{\text {exp }}$.

IR spectra are usually measured over a wide spectral range. In most parts of the spectrum no absorption bands occur and the measured transmission depends only on the real part of the index of refraction $n_{\text {film }}$ and a constant contribution to the extinction coefficient $k_{\text {film, } 0}$. These two parameters are to a first approximation considered to be constant over the measured spectral range. The first step is to fit the optical model by a variation of $n_{\text {film }}$ and $k_{\text {film, } 0}$ to the measured data of the IR spectrum in the whole range where no explicit absorption bands occur. This constant index of refraction $n_{\text {film }}$ is denoted $n_{\infty}$. Afterwards, the imaginary part $k_{\mathrm{film}}=k_{\mathrm{film}, 0}+k_{\mathrm{film}(\omega)}$ of the refractive index in the spectral range of the absorption bands can be calculated from the measured IR data using the formalism described above. It has to be taken into account, however, that $n_{\text {film }}$ is not constant in the spectral region of the absorption band, because the real part of the index of refraction $n_{\text {film }}$ is connected to the imaginary part of the index of refraction $k_{\text {film }}$ via the Kramers-Kronig relation (KKR). For the calculation of $n_{\text {film }}(\omega)$ from a known $k_{\text {film }}(\omega)$ by the KKR, it is in general necessary to know the imaginary part $k_{\text {film }}(\omega)$ of the refractive index in the whole spectral range and not only in the infrared spectral region. If $k_{\text {film }}(\omega)$ can be only measured in the infrared, the influence of the absorption in the residual spectral regions can be integrated and introduced in the KKR by $n_{\infty}$. This yields:

$$
n_{\text {film }}(\omega)=n_{\infty}+\frac{2}{\pi} P \int_{\text {infrared }} \frac{s k_{\text {film }}(s)}{s^{2}-\omega^{2}} d s
$$

where $P$ means the Cauchy principal value. With this formula the variation of $n_{\text {film }}(\omega)$ in the spectral range of the absorption bands can be calculated for a given $k_{\text {film }}(\omega)$. The self- consistent determination of $n_{\text {film }}(\omega)$ and $k_{\text {film }}(\omega)$ from the measured transmission data can 
be achieved by an iterative solution of the optical model and equation (20).

In the following a few model calculations will be shown to discuss the various optical thin- film effects occurring in IRAS measurements. The errors occurring due to a neglect of various of these thin film effects have been previously discussed by Tzolov et al. [21] for amorphous, hydrogenated silicon (a-Si:H) layers. They found that these errors can be as high as $10 \%$. All model results presented here are made for a thin film on top of a single-crystalline silicon wafer with a native silicon oxide layer of $2 \mathrm{~nm}$ thickness. The optical constants for silicon and silicon oxide are taken from Ref. 22. As examples for the optical properties of the thin films, values typical of plasma-deposited, amorphous, hydrogenated carbon films were chosen [23]. Fig. 2 shows model results for 3 film thicknesses. The solid and dashed lines are for 2 types of films without absorption $(k=0)$ with refractive indices of 1.5 and 2.0, respectively. Two points are remarkable: First, we clearly see the influence of the interference effects in the thin layer which cause the sinusoidal variation of the transmittance. The effect is the more pronounced the longer the optical path $n \times d$ is. Second, the normalized transmission is always larger than 1 . That means that the transmittance of the silicon substrate with thin film is larger than without it, and the layer works as a antireflection coating. Fig. 3 shows model results for a film with $n=1.5$ that shows absorption around 3000 $\mathrm{cm}^{-1}$. For simplicity two Gaussian lines positioned at 3000 and $3050 \mathrm{~cm}^{-1}$ each with a width $\sigma=20 \mathrm{~cm}^{-1}$ $\left(\mathrm{FWHM} \approx 47 \mathrm{~cm}^{-1}\right)$ were assumed to model $k_{\mathrm{film}}(\omega)$. The intensity of peak 1 was set to 0.1 and that of peak 2 to 0.01 . The solid and dashed lines in Fig. 3 show the model results including and neglecting the KKR, respectively. In addition, the 'background line' for an absorption free layer is shown as dotted line for the 500 and $1000 \mathrm{~nm}$ thick films. The influence of the KKR on the absorption is not dramatic, but clearly detectable. For the $500 \mathrm{~nm}$ film the KKR consistent spectrum lies for lower wave numbers above and for higher wave numbers below the KKR inconsistent spectrum. It is obvious that a consideration of the background according to the absorption free model or a KKR inconsistent analysis will cause a distortion of the $k$ spectrum. On the other hand, it will be impossible to achieve a good fit of the model to the measured spectrum if the KramersKronig relation is not observed. We can further recognize that the peak in the KKR consistent spectrum is slightly shifted to larger wave numbers compared to the KKR inconsistent model. The effects discussed for the $500 \mathrm{~nm}$ spectrum are even more pronounced in the $300 \mathrm{~nm}$ spectrum, but they are much less pronounced in the $1000 \mathrm{~nm}$ spectrum. The particular effects of the KKR vary depending on whether the absorption peak is located on the rising, falling, or flat part of the interference pattern as visible in Fig. 3. Similar effects have been presented by Tzolov et al. [21] for a-Si:H layers.

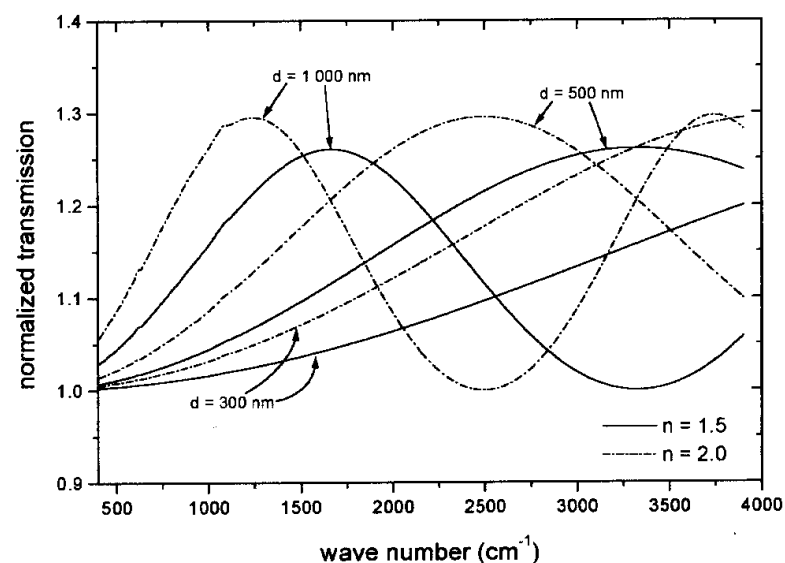

Figure 2. Model calculations for a thin transparent film on top of silicon. The simulations are made for absorptionfree layers $(k=0)$ with thicknesses of 300,500 and 1,000 $\mathrm{nm}$ assuming an refractive index of 1.5 (solid lines) and 2.0 (dashed lines). An additional inclusion of a constant extinction coefficient would cause a damping of the presented oscillatory component.

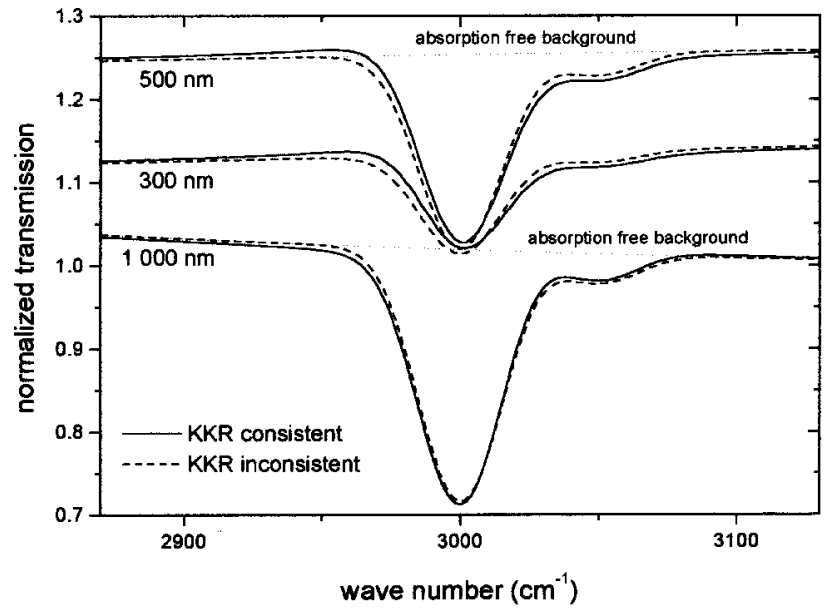

Figure 3. Model calculations for a film with an absorption structure around $3000 \mathrm{~cm}^{-1}$. For the absorption structure two peaks with Gaussian line shape were assumed. The peaks are centered at 3000 and $3050 \mathrm{~cm}^{-1}$. The width was set to $20 \mathrm{~cm}^{-1}$ and the intensities to 0.1 and 0.01 , respectively. The 'background' absorption is set to 0 . The solid lines are KKR consistent and the dashed lines are KKR inconsistent. The absorption-free background from Fig. 2 is shown as dotted line for the 500 and 1,000 $\mathrm{nm}$ spectrum.

To conclude this modeling section we can state that optical transmission spectra have to be modeled to be able to extract the complete contained information. This work is a bit tedious, but can efficiently be performed with the help of a computer program. The background in a transmission spectrum is determined by interferences in the thin film and contains thus information on the thickness and refractive index of the film. The spectra have to be calculated in a KKR consistent manner otherwise peak shifts and peak shape 
distortions may occur. The latter are particularly perturbing if small peaks next to large peaks shall be analyzed, a situation typically encountered in the analysis of the $\mathrm{CH}$ vibrational bands of a-C:H layers around $3000 \mathrm{~cm}^{-1}$. In any case where a meaningful fitting of the absorption structure shall be attempted the spectra have to be calculated KKR consistent.

\section{Experimental}

Thin films are deposited by low-temperature plasma deposition in an ECR plasma device described recently $[24,25]$. In short, microwaves of $2.45 \mathrm{GHz}$ are coupled to a plasma chamber about $20 \mathrm{~cm}$ in diameter through an aluminum oxide window. The resonance field of 87.5 $\mathrm{mT}$ is provided by external magnetic coils. The magnetic field at the coupling window is about $110 \mathrm{mT}$ decaying continuously with increasing distance from the window. The plasma is confined to a volume of about 2.7 liters by a metallic cage, to obtain a certain decoupling of the plasma production from the processes at the growing film surface. Through an aperture in the cage (35 $\mathrm{mm}$ in diameter) a plasma beam is extracted and directed onto the substrate electrode. Gas flows are measured by gas flow controllers and range from 15 to $20 \mathrm{sccm}$. The operating pressure is set to 0.2 Pa. Single-crystalline silicon is used as substrates. The substrates are mounted on a rf-driven electrode. Application of rf power leads to a dc self-bias which is varied between 0 and $-250 \mathrm{~V}$. At the low applied pressures, the plasma sheath is considered to be free collisions, so that the applied dc-self- bias plus the plasma potential of about 10 to $15 \mathrm{~V}$ yields directly the ion energy. The temperature of the substrate holder is monitored by a thermocouple.

The sample preparation is monitored in situ by realtime ellipsometry. Details of the ellipsometric set-up were presented elsewhere [26]. All ellipsometry measurements are performed at a constant wavelength of $600 \mathrm{~nm}$. Evaluation of the ellipsometry data yield the complex refractive index of the layers at $600 \mathrm{~nm}$ and the precise film thickness. Details on the deposition procedure and ellipsometric measurements for these layers are found in Ref. 25 .

After deposition the samples are investigated by high energy ion beam analysis (IBA) to determine the film stoichiometry [25]. Infrared absorption spectra are measured for films about $300 \mathrm{~nm}$ thick using a Perkin Elmer 1760X Fourier transform infrared spectrometer. The spectra are recorded in transmission at perpendicular incidence in the spectral range from 1000 to $4000 \mathrm{~cm}^{-1}$. All transmission measurements are normalized to the transmission of an uncoated silicon substrate of the same wafer. From the transmission spectra, the extinction coefficient $k$ is determined using the formalism described in Sec. II.

\section{Results}

Fig. 4 shows a series of original IRAS spectra. The films were deposited from n-butane $\left(n-\mathrm{C}_{4} \mathrm{H}_{10}\right)$. Shown are three spectra for deposition at floating potential, at $30 \mathrm{~V}$ and at $200 \mathrm{~V}$ dc self-bias. All tree layers are about $300 \mathrm{~nm}$ thick. The first thing to recognize is the continuous increase of the background with increasing wave numbers. This increase is due to the interference in the thin film as was demonstrated in Fig. 2. It compares well with the curves for $300 \mathrm{~nm}$ in Fig. 2. Superimposed to this slowly varying background we find various absorption structures. In the range from about 1300 to $1500 \mathrm{~cm}^{-1}$ we find $\mathrm{C}-\mathrm{H}$ deformation vibrations, between 1500 and $1700 \mathrm{~cm}^{-1} \mathrm{C}=\mathrm{C}$ stretching vibrations from the carbon network, and around 3000 $\mathrm{cm}^{-1} \mathrm{C}-\mathrm{H}$ stretching vibrations. In the following we will concentrate on the $\mathrm{C}-\mathrm{H}$ stretching region because the most prominent structure occurs there and this region is dominantly discussed in literature. Already from Fig. 4 it is clear, that this region is also strongly affected by the deposition conditions and, accordingly, by the resulting film structure.

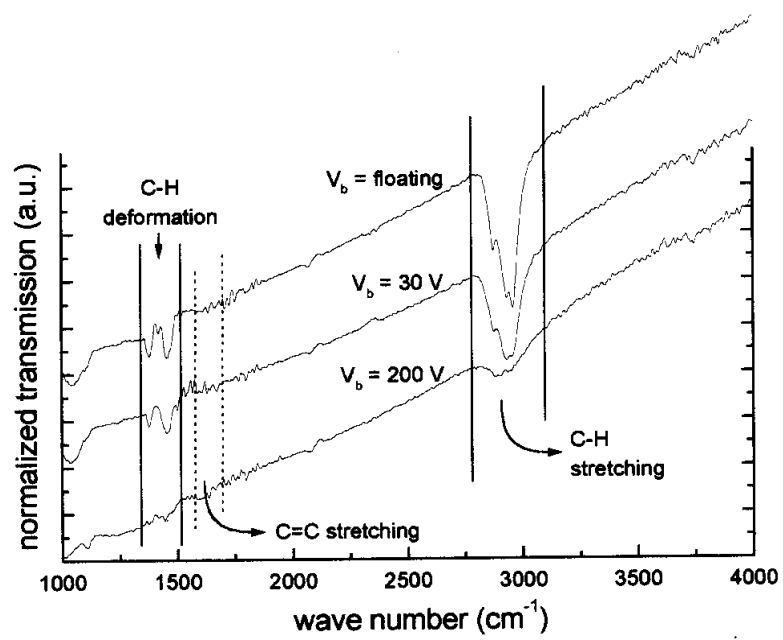

Figure 4. Original measurement data of the infrared transmission through silicon substrates coated with plasmadeposited a-C:H layers. The 3 layers are deposited from $n-\mathrm{C}_{4} \mathrm{H}_{10}$ applying different bias voltages $V_{b}$. The layers are between 250 and $300 \mathrm{~nm}$ thick. All spectra are normalized relative to the transmission of a bare silicon substrate from the same wafer. The curves are vertically offset for clarity.

Fig. 5 present the results for the optical properties of these 3 layers in the region of the $\mathrm{C}-\mathrm{H}$ stretching vibrations around $3000 \mathrm{~cm}^{-1}$. The upper part shows the real part of the refractive index $n$ and the lower part the extinction coefficient $k$. With increasing dc self-bias $V_{b}$, corresponding to increasing ion energy during deposition, the refractive index $n$ increases and $k$ decreases. For the example given, $n$ increases from about 1.49 at floating potential to 1.61 and 2.08 at -30 and $-200 \mathrm{~V}$ 
dc self-bias. The refractive index at $600 \mathrm{~nm}$, as measured by in-situ ellipsometry during deposition, shows the identical trend, but the values are somewhat higher. The corresponding values for $n(600 \mathrm{~nm})$ are $1.59,1.69$, and $2.25[25]$. The hydrogen content $[\mathrm{H} /(\mathrm{H}+\mathrm{C})]$ of the same layers as measured by ion beam analysis decreases for the same films from 0.48 (floating potential) to 0.31 $(-200 \mathrm{~V})[25]$. The variation of $\mathrm{n}$ in the region of strong absorption is clearly visible in the upper part of Fig. 5, particularly for the film deposited at floating potential $(n \approx 1.5)$. In this case, the difference between the maximum and minimum of $n$ is about $5 \%$. This variation of $n$ is due to mutual dependence of $n$ and $k$ as expressed by the Kramers-Kronig relation (Eq. 20). The variation of $n$ is less pronounced for the other two films, because there the absorption is also lower.

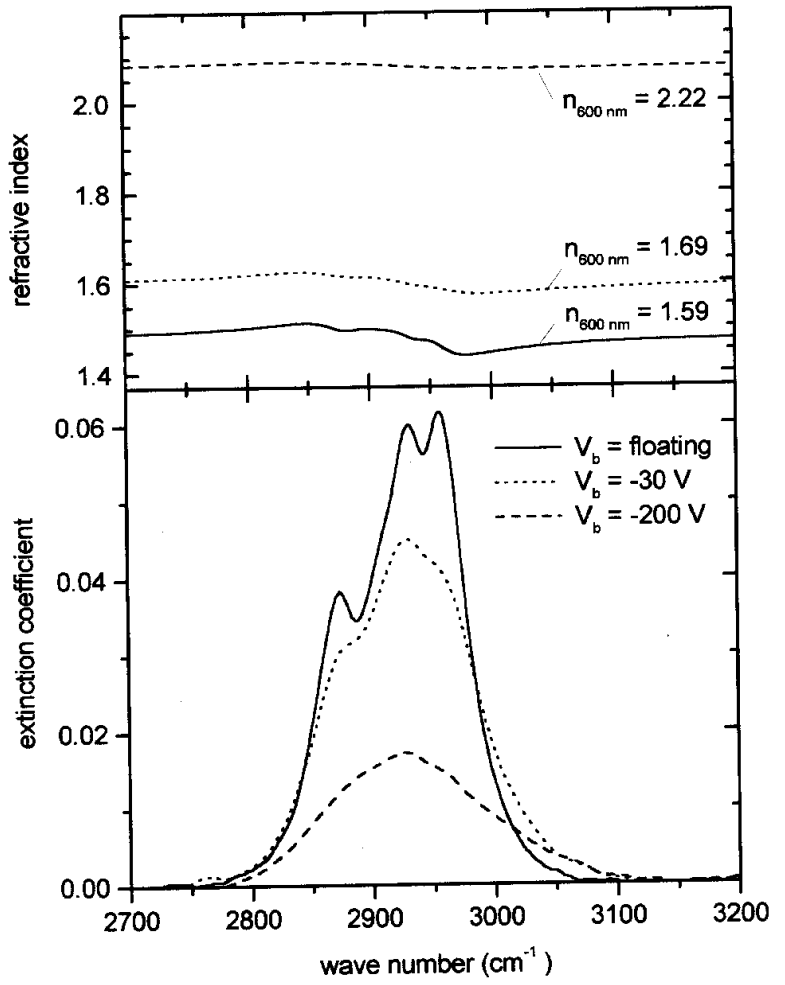

Figure 5. Real $(n)$ and imaginary $(k)$ part of the complex refractive index for the layers shown in Fig. 4.

The $k$ spectra of the three layers are distinctly different. They differ in absolute intensity as well as in shape. The general trend is that with increasing ion energy the maximum intensity and the peak integral decrease while the shape becomes broader and much less structured. The assignment of the individual $\mathrm{CH}$ bands follows the basic investigations of Dischler [27]. A very detailed investigation of the dependence of the IRAS spectra on deposition conditions was recently published by Ristein et al. [28]. The above described observation of decreasing intensity and loss of fine structure of the $\mathrm{CH}$ vibrational bands is a very general observation in the literature [25,27-29]. A thorough discussion of the change of the IR absorption structure would lead too far here and will be published elsewhere [30]. We can, however, summarize that the observed changes reflect the changes of the microstructure of the deposited layers. With increasing ion energy the density of $s p^{3}-\mathrm{CH}_{3}$ groups, which contribute very strongly to the observed structure, decreases while the density of $s p^{3}-\mathrm{CH}_{2}, s p^{3}-\mathrm{CH}$, and $s p^{2}-\mathrm{CHx}$ groups increases. The infrared absorption cross sections (often also called dipole strength) for the latter groups, in particular for the $s p^{2}$-related bands, is significantly lower than for the $s p^{3}-\mathrm{CH}_{3}$ groups $[9,28]$. Together with the integral decrease of the hydrogen content and the increase of $s p^{2}$ character [23] this accounts for the decrease of the $\mathrm{CH}$ vibrational band intensity. In a recent study of the influence of hydrocarbon source gas on the properties of plasma- deposited thin films, we found a strong correlation of all investigated physical properties of the layers [25]. In particular, the density, the refractive index, and the hydrogen content exhibit a very strong correlation. A comparison of the integral over the $\mathrm{CH}$ vibrational band with the other film properties has shown that the IR $k$ spectra of the a-C:H layers are also strongly correlated to the other film parameters and can be used as a fingerprint of the system as demonstrated in Fig. 5. Soft, polymerlike hydrocarbon layers posses a high hydrogen content (up to more than $60 \%)$, a low refractive index $(n \approx 1.5)$, a low density (down to values below $1 \mathrm{~g} \mathrm{~cm}^{-3}$ ) [23,25], and are characterized by a well structured IR absorption band with maximum values for $k>0.06$ around 3000 $\mathrm{cm}^{-1}$. On the contrary, hard amorphous hydrogenated carbon layers ('diamond-like carbon') posses a low hydrogen content (typically around $30 \%$ ), high refractive index $(n>2)$, high density $\left(>1.8 \mathrm{~g} \mathrm{~cm}^{-1}\right)$ and exhibit a structureless, broad IR absorption band with relative low intensity $(k<0.02)$.

\section{Conclusions}

This article discussed the infrared analysis of thin films choosing the example of plasma- deposited, amorphous, hydrogenated carbon layers. First the framework for the optical analysis of thin films was presented. The main characteristic of thin film optics is the occurrence of interference effects due to the coherent superposition of light multiply reflected at the various internal and external interfaces of the optical system. These interference effects lead to a sinusoidal variation of the transmitted and reflected intensity and are commonly present in published IRAS spectra. They contain information on the refractive index and the film thickness. A consequence of these interference effects is that the Lambert-Beer law is, in general, not applicable for the determination of the absorption coefficient of thin films.

It was further shown, that the mutual interdepen- 
dence of real and imaginary part of the complex refractive index as expressed by the Kramers-Kronig relation leads to observable changes of the transmission and reflection spectra. A neglect of these effects in the data evaluation will cause a distortion of the peak shape and a slight shift of the peak position. If a meaningful analysis of the absorption peaks shall be attempted, e.g., a peak decomposition, it is indispensable to model the transmission (or reflection spectra) using the full formalism including the Kramers-Kronig relation.

Second, infrared absorption spectra and the resulting $k$ spectra in the range of the $\mathrm{CH}$ vibrational bands around $3000 \mathrm{~cm}^{-1}$ were presented. The shape and the total intensity of the peak are quite sensitive to the film structure. Soft, polymerlike hydrocarbon layers are characterized by a well structured, intense IR absorption band, while hard, amorphous, hydrogenated carbon layers exhibit a structureless, broad IR absorption band with relative low intensity. The integral of the $k$ spectra of the $\mathrm{CH}$ vibrational band of a-C:H layers around $3000 \mathrm{~cm}^{-1}$ is strongly correlated to the other physical parameter of the layers such as density, hydrogen content, and refractive index. Due to the strong sensitivity of the $k$ spectra of the $\mathrm{CH}$ vibrational band to the film structure, they can be considered as fingerprint for the type of a-C:H film.

\section{References}

[1] A large number of textbooks on infrared spectroscopy exists in many languages. The following are only an exemplary selection: L.J. Bellamy, Advances in Infrared Group Frequencies, Methuen, London, 1968. N.B. Colthup, L.H. Daly, and S.E. Wiberly, Introduction to Infrared and Raman Spectroscopy, Academic Press, Boston, 1990.

[2] H. Günzler and H.M. Heise, IR-Spektroskopie: Eine Einführung, 3. Ed., VCH Verlag, Weinheim, 1996.

[3] N.J. Harrick, Internal Reflection Spectroscopy, Wiley, New York, 1967.

[4] E.D. Palik (Ed.), Handbook of Optical Constants of Solids II, Academic Press, Boston, 1991.

[5] M.H. Brodsky, M. Cardona, and J.J. Cuomo, Phys. Rev. B 16, 3556 (1977).

[6] A.A. Langford, M.L. Fleet, B.P. Nelson, W.A. Lanford, and N. Maley, Phys. Rev. B 45, 13367 (1992).

[7] A. Grill and V.V. Patel, Appl. Phys. Lett. 60, 2089 (1992).

[8] W. Jacob and W. Müller, Appl. Phys. Lett. 63, 1771 (1993).

[9] W. Jacob and M. Unger, Appl. Phys. Lett. 68, 475 (1996).
[10] H. Gobrecht (ed.), Bergmann Schaefer Lehrbuch der Experimental Physik Bd. III: Optik, 8. Ed., Walter de Guyter, Berlin, 1987.

[11] O. Stenzel, Das Dünnschichtspektrum, Akademie Verlag, Berlin 1996.

[12] M. Born and E. Wolf, Principles of Optics, Pergamon Press, New York, 5th Ed., 1975.

[13] H.A. Macleod, Thin-Film Optical Filters, Adam Hilger LTD, London, 1969.

[14] O. Stenzel and A. Stendal, Optical Properties in Wiley Enciclopedia of Electrical and Electronics Engineering Vol. 15, J.G. Webster (ed.), John Wiley and Sons, New York, 1999, p. 327- 336.

[15] B. Harbecke, B. Heinz, V. Offermann, and W. Thei $\beta$, Far-Infrared Spectroscopy in Optical Characterization of Epitaxial Semiconductor Layers, G. Bauer and W. Richter (eds.), Springer, Berlin 1996, pp. 203.

[16] P. Grosse, B. Harbecke, B. Heinz, R. Meyer, M. Offenberg, Appl. Phys. A 39, 257 (1986).

[17] B. Harbecke, B. Heinz, P. Grosse, Appl. Phys. A 38, 263 (1985).

[18] B. Harbecke, Appl. Phys. B 39, 165 (1986).

[19] R.M.A. Azzam and N.M. Bashara, Ellipsometry and Polarized Light, Elsevier Science Publishers, Amsterdam 1977.

[20] U. Rossow and W. Richter, Spectroscopic Ellipsometry in Optical Characterization of Epitaxial Semiconductor Layers, G. Bauer and W. Richter (eds.), Springer, Berlin 1996, pp. 68.

[21] M.B. Tzolov, N.V. Tzenov, and D.I. DimovaMalinovska, J. Phys. D: Appl. Phys. 26, 111 (1993).

[22] E.D. Palik, (ed.), Handbook of Optical Constants of Solids, Academic Press, Orlando, 1985.

[23] W. Jacob, Thin Solid Films 326, 1 (1998).

[24] B. Landkammer, A. von Keudell, and W. Jacob, J. Nucl. Mater. 264, 48 (1999).

[25] T. Schwarz-Selinger, A. von Keudell, and W. Jacob, J. Appl. Phys. 86, 3988 (1999).

[26] W. Fukarek and A. von Keudell. Rev. Sci. Instrum. 66, 2029 (1995).

[27] B. Dischler, in Amorphous Hydrogenated Carbon Films EMRS Symp. Proc. Vol. 17, P. Koidl and P. Oelhafen (eds.), Les Editiones de Physique, Paris 1987, p. 198.

[28] J. Ristein, R.T. Stief, L. Ley, and W. Beyer, J. Appl. Phys. 84, 3836 (1998).

[29] A. von Keudel and W. Jacob, J. Vac. Sci. Technol. A15, 402 (1997).

[30] T. Schwarz-Selinger, A. von Keudell, and W. Jacob, to be published. 